\title{
Anabases
}

ANABASES Traditions et réceptions de l'Antiquité

$4 \mid 2006$

Varia

\section{E. TEYSSIER et B. LOPEZ, Gladiateurs. Des sources à l'expérimentation}

Robert Sablayrolles

\section{OpenEdition}

Journals

Édition électronique

URL : http://journals.openedition.org/anabases/2873

DOI : 10.4000/anabases. 2873

ISSN : 2256-9421

Éditeur

E.R.A.S.M.E.

Édition imprimée

Date de publication : 1 octobre 2006

Pagination : $324-326$

ISSN : 1774-4296

Référence électronique

Robert Sablayrolles, «E. TeYssier et B. Lopez, Gladiateurs. Des sources à l'expérimentation », Anabases [En ligne], 4 | 2006, mis en ligne le 01 mai 2012, consulté le 22 septembre 2020. URL : http:// journals.openedition.org/anabases/2873 ; DOI : https://doi.org/10.4000/anabases.2873

Ce document a été généré automatiquement le 22 septembre 2020.

(c) Anabases 


\title{
E. TEYSSIER et B. LOPEZ, Gladiateurs. Des sources à l'expérimentation
}

\author{
Robert Sablayrolles
}

\section{RÉFÉRENCE}

E. TEYSSIER et B. LOPEZ, Gladiateurs. Des sources à l'expérimentation, Paris, éd. Errance, 2005, $192 \mathrm{p}$.

29 euros / ISBN 2877723151.

1 L'archéologie expérimentale est à la mode, ce qui, comme la langue d'Ésope, est la meilleure et la pire des choses au monde. Le principal atout de l'archéologie expérimentale est qu'elle allie expériences scientifiques de reconstitution, qui permettent aux chercheurs de tester leurs hypothèses dans une démarche de science expérimentale, et spectacle grand public, qui offre à un très large cercle d'amateurs ou de profanes une image vivante de la réalité antique, marquée du sceau de l'authenticité par la présence d'auteurs reconnus comme des spécialistes. Le risque majeur est que le spectacle prenne le pas sur la recherche expérimentale, comme dans nombre de «documentaires fictions» dont les télévisions soucieuses d'audimat abreuvent un public auquel est servi généreusement ce qu'il s'attend à trouver.

2 Eric Teyssier, agrégé d'histoire et maître de conférences à l'université de Nîmes, et Brice Lopez, professeur d'arts martiaux et troisième dan en jiu-jitsu, ont uni leurs compétences dans une démarche originale qui a consisté à appliquer les principes de l'archéologie expérimentale à un domaine riche en interrogations scientifiques comme en clichés ressassés : celui de la gladiature dans le monde romain. Malgré le nombre considérable de sources antiques relatives au phénomène, de la littérature à l'iconographie, de l'épigraphie aux vestiges archéologiques les plus divers, de nombreuses interrogations restent en effet sans réponse assurée. Les remarquables travaux fondateurs de L. Robert et de G. ville et les synthèses récentes de la science italienne, comme les corpus épigraphiques de P. sabbatini Tumolesi, ou les colloques 
Spectacula organisés et publiés au Musée Henri Prades de Lattes par Chr. Landes - et non pas par E. Bouley comme l'indique la bibliographie de l'ouvrage d'E. Teyssier et B. Lopez (p. 153)- ont souligné les incertitudes sur les identifications de différents types de gladiateurs et n'ont laissé qu'une faible place aux aspects techniques du combat.

Pour combler ces lacunes, E. Teyssier et B. Lopez sont partis des sources iconographiques, archéologiques, épigraphiques et littéraires pour reconstituer les équipements des gladiateurs antiques, identifier les différents types de combattants, et organiser une troupe de gladiateurs, dont la fonction est à la fois de construire et de tester des hypothèses sur les pratiques techniques et d'offrir des spectacles éducatifs autant que distrayants, dans lesquels sont détaillées et expliquées les diverses formes d'assauts, de défenses et d'affrontements. De ce point de vue, le livre apporte un éclairage nouveau et assure un certain nombre d'acquis. Il est très largement illustré (deux cents figures) de photographies en couleur d'objets, d'inscriptions ou de représentations antiques, mais aussi et surtout de gladiateurs modernes dans les différentes postures et gestes de leur art. De façon convaincante, grâce aux images (parfois trop nombreuses cependant) et à leurs légendes très détaillées, les auteurs mettent en relief, et c'est une nouveauté, le rôle fondamental et central du bouclier dans l'affrontement. Cette conclusion, solidement étayée dans une analyse qui souligne bien la différence entre gladiature et escrime, donne une nouvelle dimension au conflit traditionnel qui, dans l'Antiquité, opposait scutarii, partisans de gladiateurs au bouclier long et lourdement armés, et parmularii, amateurs des gladiateurs légers au bouclier court ou rond. De même, les auteurs offrent, par le croisement des sources, des hypothèses d'identification intéressantes : pour le scissor et sa main droite équipée d'un tranchant en demi-lune ou pour l'hoplomaque dont la lance permet de comprendre le trait grinçant du poète Martial qui, parlant d'un oculiste devenu hoplomaque, disait de lui qu'il pratiquait son nouveau métier avec le même art que l'ancien (Martial, Épigrammes, VIII, 74).

4 Cette louable et remarquable entreprise d'archéologie expérimentale aurait dû, cependant, se limiter à ce qui était son but essentiel et originel : les questions de techniques de combat. L'approche de la vie quotidienne des gladiateurs et, plus largement, du phénomène de la gladiature dans la société romaine souffre d'approximations. Ainsi, par exemple, il n'est pas nécessaire d'aller imaginer, dans l'opposition entre le rétiaire, qui combat sans équipement défensif, et son adversaire plus lourdement armé, le symbole d'une opposition sociale entre pauvres et riches, qui aurait séduit une partie du public (p. 58). Le goût des spectateurs pour ce type d'opposition venait tout simplement, comme les auteurs l'écrivent eux-mêmes un peu plus loin (p. 60 et 67), de l'affrontement de combattants aux atouts opposés.

5 Une relative incertitude paraît peser sur la condition juridique que les auteurs prêtent aux gladiateurs. Parlant du secutor Urbicus (CIL, V, 5933 - et non pas, 05, 5933, mode de référence adopté dans l'ensemble de l'ouvrage), ils écrivent: «La seule mention du nom permet de déduire que ce combattant n'est pas un citoyen romain» (p. 136). C'est oublier que le gladiateur, juridiquement infamis, est au ban de la société et ne saurait donc être, en aucun cas, un citoyen. Urbicus peut être un pérégrin libre, sans être ni citoyen ni affranchi ni esclave. Les citoyens qui s'engageaient dans la gladiature perdaient par le fait même leur dignité juridique de citoyen. Il n'est pas vrai pour autant que « dans l'arène, quelles que soient leurs origines, les deux combattants ont le statut d'esclave » (p. 131). Leurs conditions de vie, qui les enchaînaient, par la formule 
de l'auctoratio, au laniste, les rapprochaient certes des esclaves dépendants de leurs maitres, mais, à la différence des esclaves, les gladiateurs libres (anciens citoyens ou anciens pérégrins) restaient des personnes possédant une personnalité juridique reconnue, qui leur donnait, notamment, un droit de propriété et un pouvoir de léguer par testament que n'ont jamais eus les esclaves.

6 Se fondant sur des statistiques aux bases quantitatives bien faibles (les affiches peintes de Pompéi), les auteurs supposent que seulement $10 \%$ des combats se terminaient par la mort. C'est faire peu de cas de la dimension tragique du spectacle, qui n'était pas seulement un sport, mais une cérémonie où la société, rangée par ordre hiérarchique dans la cauea, venait contempler son pouvoir d'organiser la mort en direct, et donc le destin. La recherche de l'économie, prêtée dans ce domaine aux évergètes, est contradictoire avec la pratique même de l'évergétisme, où le donateur devait montrer sa libéralité et même si possible surprendre grâce à elle, par un don gratuit, allant audelà de ce que le public pouvait imaginer. Il est surprenant, sur ce chapitre, qu'aucune mention ne soit faite du senatus-consulte de l'époque de Marc Aurèle (CIL, II, 6278) réglementant très strictement la fiscalité des ludi et les contrats financiers passés entre lanistes et organisateurs des jeux, réaction aux pratiques visiblement excessives en la matière. La découverte archéologique récente d'une nécropole de gladiateurs à Éphèse, étudiée par l'Institut Archéologique Autrichien et l'université de Médecine de Vienne, montre que la plupart des corps, avaient subi des blessures et que les combattants avaient été achevés par un coup porté à la tête, fracassée par un instrument contondant. Certes, là encore, le chiffre est faible et la statistique limitée à une nécropole particulière, mais il est plus élevé que celui des affiches de Pompéi, qui ne reflètent, elles aussi, qu'une réalité très partielle : aucune conclusion ne pourra jamais être solidement établie en la matière et toute affirmation péremptoire est forcément infondée. En termes de vraisemblance, les seules que l'on puisse s'autoriser, les considérations sur la mise en scène " live » du tragique et sur les lois de l'évergétisme suggèrent des proportions de décès au combat autrement élevées.

7 Il est regrettable que ces approximations et une écriture pas toujours maitrisée ou une relecture trop rapide - quelques coquilles comme armentarium, étable, au lieu d' armamentarium, armurerie, p. 7 ; «Se faisant » pour «ce faisant », p. 31 ; «Un matériel archéo-compatible», p. 45... - aient quelque peu terni l'image d'une entreprise novatrice, qui a su allier, par son essence même, recherche scientifique et valorisation de la recherche et qui a analysé de façon convaincante certains aspects techniques de la gladiature.

\section{AUTEURS}

\section{ROBERT SABLAYROLLES}

Université Toulouse II - Le Mirail

sablayro@univ-tlse2.fr 